$\underset{\text { ISSN 109-4300 }}{\text { Entropy }}$

www.mdpi.org/entropy

\title{
Modified Feynman ratchet with velocity-dependent fluctuations
}

\author{
Jack Denur \\ Electric \& Gas Technology, Inc.; 13636 Neutron Road; Dallas, Texas 75244-4410. \\ E-mail:pd1@ticnet.com, elgt@elgt-amti.com \\ Received: 2 July 2003/Accepted 10 December 2003/ Published 15 March 2004
}

\begin{abstract}
The randomness of Brownian motion at thermodynamic equilibrium can be spontaneously broken by velocity-dependence of fluctuations, i.e., by dependence of values or probability distributions of fluctuating properties on Brownian-motional velocity. Such randomness-breaking can spontaneously obtain via interaction between Brownian-motional Doppler effects - which manifest the required velocity-dependence - and system geometrical asymmetry. A nonrandom walk is thereby spontaneously superposed on Brownian motion, resulting in a systematic net drift velocity despite thermodynamic equilibrium. The time evolution of this systematic net drift velocity - and of velocity probability density, force, and power output - is derived for a velocity-dependent modification of Feynman's ratchet. We show that said spontaneous randomness-breaking, and consequent systematic net drift velocity, imply: bias from the Maxwellian of the system's velocity probability density, the force that tends to accelerate it, and its power output. Maximization, especially of power output, is discussed. Uncompensated decreases in total entropy, challenging the second law of thermodynamics, are thereby implied.
\end{abstract}

PACS numbers: 05.40.Jc, 05.40.Fb, 02.50.Ga, 05.20.Dd, 02.50.Ey, 02.50.Cw.

AMS numbers: 60J10, 28D20, 60G50, 82B41, 82B40, 82B05.

Keywords: second law of thermodynamics, spontaneous momentum flow, Feynman ratchet, velocity-dependent fluctuations, Doppler effect, nonrandom walk.

\section{Velocity-dependent modified Feynman ratchet}

The Zhang [1] formulation of the second law of thermodynamics (second law) states that no spontaneous momentum flow is possible in an isolated system. By spontaneous, it is meant [1]: not merely (a) sustaining, i.e., permanent; but also (b) robust, i.e., capable of withstanding dissipation, of surviving disturbances, and of generating (regenerating) itself if initially nonexistent (if destroyed). The Zhang [1] formulation of the second law implies that, at thermodynamic equilibrium (TEQ), not even merely 
sustaining momentum flow is possible, i.e., that no systematic motion - most generally, no systematic process - is possible at TEQ: Systematic processes generated and maintained spontaneously despite $T E Q$ violate the second law; by contrast, systematic merely sustaining, i.e., nonrobust and nondissipative — and hence nonspontaneous - processes do not violate the second law, but merely imply that TEQ has not been completely realized [1,2]. [Given any irreversibility (e.g., friction), (nonspontaneous) merely sustaining processes lose even their sustainability - they become nonrobust and dissipative - their negentropy and free energy are lost, and TEQ is completely realized [1,2].]

Feynman's classic ratchet and pawl [3] elucidates the Zhang [1] formulation of the second law. Recently, various formulations of the second law have been challenged, mainly in the quantum regime [4-7], but also classically [4,8].

In this paper [9], we show that velocity-dependent fluctuations (but not fluctuations in general) challenge the second law in the classical regime. (Our challenge may also obtain in the quantum regime, but this aspect is not studied herein.) Our challenge is most self-evident with respect to the Zhang [1] formulation of the second law, but a challenge to the Zhang [1] formulation of the second law is also a challenge to all other formulations thereof. Feynman's ratchet [3] is modified to the minimum extent necessary to ensure that velocity-dependence of fluctuations can spontaneously break the randomness of its Brownian motion at TEQ - spontaneously superposing a nonrandom walk on its Brownian motion and hence challenging the second law. This minimally-modified Feynman ratchet, illustrated in Fig. 1, will now be described.

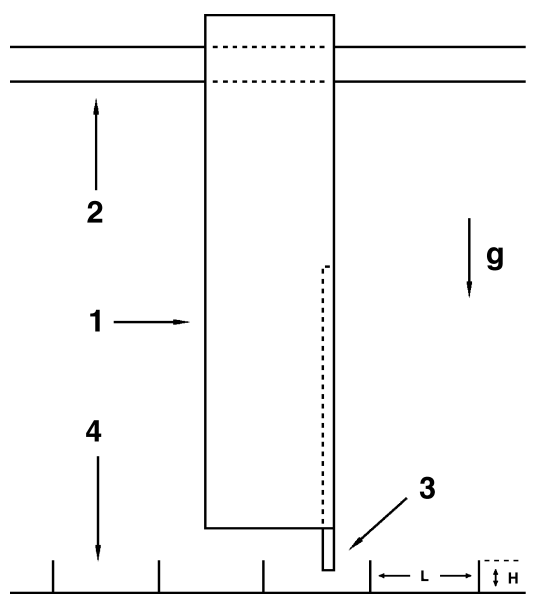

Figure 1. Modified Feynman ratchet with velocity-dependent fluctuations.

In the right-handed Cartesian coordinate system of Fig. 1 , the $+X,+Y$, and $+Z$ directions are to the right, into the page, and upwards, respectively. The Brownian motion of the disk 1 of mass $m^{\prime}$ (shown edge-on in Fig. 1) is constrained to be $X$-directional by the frictionless guide 2. The pawl 3 of mass $m$ (whose lower tip protrudes below the disk in Fig. 1) is in a vertical groove within the $+X$ disk face, wherein - in addition to its $X$-directional Brownian motion in lockstep with the disk as part of the combined disk-and-pawl system (DP) - it also has $Z$-directional Brownian motion relative to the disk per se. The DP's total mass is $M=m^{\prime}+m \gg m$. Each peg 4 is of $Z$-directional height $H$, and is separated from adjacent pegs by $X$-directional distance $L$. The pawl's altitude $Z$ is the vertical distance of its undersurface above the $Z=0$ level at the floor of the peg row 4 , and is restricted to $Z \geq Z_{\min }$ $\left(0<Z_{\min }<H\right)$ by a stop [10] within the $+X$ disk face. The net peg height is thus $H_{\text {net }}=H-Z_{\min }$ 
$\left(0<H_{\text {net }}<H\right)$. The DP, and the entire system, is at TEQ with equilibrium blackbody radiation (EBR) at temperature $T$. $L$ can easily be small enough so that changes in the DP's $X$-directional Brownianmotional velocity $V$ occur, essentially, only at pawl-peg bounces, and not via DP-EBR $X$-directional momentum exchanges between pawl-peg bounces [11] (see the Appendix); yet (for simplicity) large compared with the combined pawl-plus-peg $X$-directional thickness. (The frictionless guide 2, of course, has no effect on $V$.) A uniform gravitational field $g$ is attractive downwards (in the $-Z$ direction). The $V=0$ rest frame - wherein (a) the frictionless guide 2 and peg row 4 are fixed and (b) the EBR at temperature $T$ is isotropic - is (for simplicity) taken as that of $g$ 's source [of mass $\gg M$ (or even $\ggg M)]$.

Corresponding to $V$, to first order in $V / c$, Doppler-shifted EBR at temperature [12]

$$
T_{ \pm}(V, \alpha)=T\left(1 \pm \frac{V \cos \alpha}{c}\right)
$$

impinges on the $\pm X$ disk face at angle $\alpha$ from the $\pm X$ direction - at a rate proportional both to the differential solid angle $2 \pi \sin \alpha d \alpha$ and, by Lambert's cosine law, to $\cos \alpha$ [12]. \{The pawl, being in the $+X$ disk face, "sees" EBR impinging - as per the immediately preceding sentence [including (1)] with the + signs - only from directions with $+X$ components (except for its lower tip - of negligible size compared with the entire pawl even at maximum tip protrusion, i.e., even at $Z=Z_{\min }$ - when said tip protrudes below the disk). $\}$ Averaging over the range $0 \leq \alpha \leq \pi / 2$ [12],

$$
T_{ \pm}(V)=\left\langle T_{ \pm}(V, \alpha)\right\rangle=\frac{\int_{0}^{\pi / 2} T\left(1 \pm \frac{V \cos \alpha}{c}\right) \sin \alpha \cos \alpha d \alpha}{\int_{0}^{\pi / 2} \sin \alpha \cos \alpha d \alpha}=T\left(1 \pm \frac{2 V}{3 c}\right) .
$$

The DP's thermal response time is sufficiently short that $T_{+}(V)\left[T_{-}(V)\right]$ is the temperature, corresponding to $V$ having a given value, of the $+X$ disk face (including the pawl) itself [11] [of the $-X$ disk face itself [11]], and not merely of Doppler-shifted EBR "seen" thereby [12]. (See the Appendix.)

The stop [10] within the $+X$ disk face - and hence itself [11] at temperature, corresponding to $V$ having a given value, of $T_{+}(V)[11,12]$ - restricts the pawl's altitude to $Z \geq Z_{\min }$ : this prevents mechanical thermal contact [although not radiative thermal contact (which is negligible)] between the floor of the peg row - at elevation $Z=0$ and temperature $T$ - and the pawl's undersurface [11c]. The pawl's thermal isolation within the $+X$ disk face is thereby improved - helping to ensure that $T_{+}(V)$ is the temperature, corresponding to $V$ having a given value, of the pawl itself [11]. (See the Appendix.)

In accordance with the Boltzmann distribution, applying (2) with the + signs, and defining $A \equiv$ $m g H_{\text {net }} / k T$ : the conditional probability [13] $P(Z>H \mid V)$ that the pawl, of weight $m g$, can attain sufficient altitude $Z>H$ to jump the pegs - and hence not to impede the DP's $X$-directional Brownian motion - given $V$, is

$$
\begin{aligned}
P(Z>H \mid V) & =\exp \left[-m g H_{\mathrm{net}} / k T_{+}(V)\right]=\exp \left\{-m g H_{\mathrm{net}} /\left[k T\left(1+\frac{2 V}{3 c}\right)\right]\right\} \\
& \equiv \exp \left[-A /\left(1+\frac{2 V}{3 c}\right)\right]=\left(1+\frac{2 A V}{3 c}\right) e^{-A} .
\end{aligned}
$$

The last step of (3) is correct to first order in $V / c$, and is justified because $|V| \ll c$ for all values of $|V|$ that have nonnegligible probabilities of being equaled or exceeded. 
By (3), $P(Z>H \mid V)$ is slightly greater when $V>0$ than when $V<0$. Hence, despite TEQ, the velocity-dependence of $P(Z>H \mid V)$ spontaneously superposes a nonrandom walk in the $+X$ (Forward) direction on the DP's Brownian motion - challenging the second law.

Note that $T_{ \pm}(V, \alpha), T_{ \pm}(V), Z$, and $P(Z>H \mid V)$ manifest velocity-dependent fluctuations. By contrast, $T, H, Z_{\min }, H_{\text {net }}=H-Z_{\min }, L, g, m^{\prime}, m, M=m^{\prime}+m \gg m$, and $A \equiv m g H_{\text {net }} / k T$ are parameters, fixed in any one given (thought) experiment.

\section{Markovian time evolution}

By (3), we have, to first order in $|V| / c$, for the conditional probabilities [13] $F$ and $R$ of $Z>H$ obtaining given DP Brownian motion in, respectively, the Forward or $+X$ direction at $V=+|V|$ and Reverse or $-X$ direction at $V=-|V|$,

$$
F \equiv P(Z>H|V=+| V \mid) \equiv P(>\mid+)=\left(1+\frac{2 A|V|}{3 c}\right) e^{-A}
$$

and

$$
R \equiv P(Z>H|V=-| V \mid) \equiv P(>\mid-)=\left(1-\frac{2 A|V|}{3 c}\right) e^{-A}
$$

respectively. The states $Z>H, Z<H, V=+|V|>0$, and $V=-|V|<0$ are denoted as $>$, $<$, + , and - , respectively. (Since $Z$ and $V$ are continuous random variables, the point values $Z=H$ and $V=|V|=0$ each has zero probability measure of occurrence.) Given $V= \pm|V|$, immediately preceding any pawl-peg interaction, the DP is in one of the four states $>+,>-,<+$, or $<-$; the former two states implying that this interaction will be a pawl-over-peg jump, and the latter two that it will be a pawl-peg bounce. Immediately following a jump (bounce), $\operatorname{sgn} V$ is unchanged (reversed).

We now study our system's time evolution, given $V= \pm|V|$, in discrete time-steps of $\Delta t=L /|V|$ that separate consecutive pawl-peg interactions, with time $N$ immediately preceding the $(N+1)$ st pawlpeg interaction [11d]. If a quantity $Q$ or an average thereof is time-dependent, then its value at time $N$ is indicated via a subscript $N$. Let $\langle Q\rangle_{N}\left(\langle\langle Q\rangle\rangle_{N}\right)$ denote the expectation value at time $N$ of a quantity $Q$ over any one given $\pm|V|$ pair $\left(\langle Q\rangle_{N}\right.$ itself averaged over all $\left.|V|\right)$. [Note: All averages in this paper are, in this wise, either over any one given $\pm|V|$ pair or over all $|V|$, except - with denotation via enclosure within single angular brackets - (a) the average $\left\langle T_{ \pm}(V, \alpha)\right\rangle$ over $\alpha$ in (2), and (b) two of the averages in the Appendix.]

TEQ, i.e., maximum initial total entropy, implies that initially, at $N=0$,

$$
\begin{aligned}
& P(+)_{0}=P(-)_{0}=\frac{1}{2} \\
\Longleftrightarrow \quad & \langle V\rangle_{0}=|V|\left[P(+)_{0}-P(-)_{0}\right]=0 \Longrightarrow\langle\langle V\rangle\rangle_{0}=0 .
\end{aligned}
$$

The expression in (6) for $\langle V\rangle_{0}$ is true for all $\pm|V|$ pairs, hence implying that for $\langle\langle V\rangle\rangle_{0}$.

Given $V= \pm|V|$ and $P(+)_{N}+P(-)_{N}=P(>\mid+)+P(<\mid+)=P(>\mid-)+P(<\mid-)=1$, said time evolution is a two-state discrete-time Markov chain [14] with (a) states + and - , and (b) the following conditional transition probabilities: $P\left[(+)_{N} \mid(+)_{N-1}\right]=P(>\mid+)=F, P\left[(-)_{N} \mid(-)_{N-1}\right]=$ 
$P(>\mid-)=R, P\left[(-)_{N} \mid(+)_{N-1}\right]=P(<\mid+)=1-F$, and $P\left[(+)_{N} \mid(-)_{N-1}\right]=P(<\mid-)=1-R$. For all $N \geq 0$, we obtain [14]

$$
\begin{aligned}
P( \pm)_{N} & =\left\{1 \pm(F-R)\left[1-(F+R-1)^{N}\right] /(2-F-R)\right\} / 2 \\
& =\left\{1 \pm(2|V| / 3 c) A\left[1-\left(2 e^{-A}-1\right)^{N}\right] /\left(e^{A}-1\right)\right\} / 2
\end{aligned}
$$

(4) and (5) being applied in the second step of (7). Applying (7), (6), (4), and (5) yields, for all $N \geq 0$,

$$
\begin{aligned}
\langle V\rangle_{N} & =|V|\left[P(+)_{N}-P(-)_{N}\right] \\
& =|V|(F-R)\left[1-(F+R-1)^{N}\right] /(2-F-R) \\
& =\left(2 V^{2} / 3 c\right) A\left[1-\left(2 e^{-A}-1\right)^{N}\right] /\left(e^{A}-1\right) .
\end{aligned}
$$

By (8), $\langle V\rangle_{N}$ is antisymmetric in $F$ and $R$; hence, taking $F \geq R \Longrightarrow\langle V\rangle_{N} \geq 0$ as per (4), (5), (6), and (8) — and throughout this paper — entails no loss of generality. The equality $F=R \Longrightarrow$ $\langle V\rangle_{N}=0$ obtains only given: (a) the point value $V=|V|=0$, which has zero probability measure of occurrence; and/or (b) $N=0$. Given $|V|>0$ and $N \geq 1$, the strict inequality $F>R \Longrightarrow\langle V\rangle_{N}>0$ despite TEQ challenges the second law.

Applying (8) and (6) in the first line of (9), and the paragraph immediately following (8) in the second, a simpler alternative to $(7)$ is

$$
\begin{aligned}
& P( \pm)_{N}=P(V= \pm|V|)_{N}=\frac{1}{2}\left(1 \pm \frac{\langle V\rangle_{N}}{|V|}\right)=P(V)_{0}\left(1 \pm \frac{\langle V\rangle_{N}}{|V|}\right) \\
\Longrightarrow & P(V)_{N}=P(V)_{0}\left(1+\frac{\langle V\rangle_{N}}{V}\right) .
\end{aligned}
$$

Considering any one given $\pm|V|$ pair, $P(V)_{0}=P(+)_{0}=P(-)_{0}=\frac{1}{2}$. By contrast, considering all $\pm|V|$ pairs, i.e., all $V$, and hence also all $|V|, P(V)_{0}=P(V)_{\mathrm{mw}}=(M / 2 \pi k T)^{1 / 2} \exp \left(-M V^{2} / 2 k T\right) \Longrightarrow$ $P(|V|)_{0}=P(|V|)_{\mathrm{mw}}=2 P(V)_{0}=2 P(V)_{\mathrm{mw}}=(2 M / \pi k T)^{1 / 2} \exp \left(-M V^{2} / 2 k T\right), P(V)_{\mathrm{mw}}$ $\left(P(|V|)_{\text {mw }}\right)$ being the one-dimensional Maxwellian probability density of $V(|V|)$.

By Newton's second law, (8), (4), and (5), the force $f$ that tends to accelerate the DP in the $+X$ direction and DP power output $P^{*}$ (not to be confused with probability $P$ ) at the $N \longrightarrow N+1$ transition, i.e., at the $(N+1)$ st pawl-peg interaction, averaged over any one given $\pm|V|$ pair, are, respectively,

$$
\begin{aligned}
\langle f\rangle_{N+\frac{1}{2}} & =M\left(\langle V\rangle_{N+1}-\langle V\rangle_{N}\right) / \Delta t=M\left(\langle V\rangle_{N+1}-\langle V\rangle_{N}\right) /(L /|V|) \\
& =\frac{M V^{2}(F-R)(F+R-1)^{N}}{L}=\frac{4 M|V|^{3} A e^{-A}\left(2 e^{-A}-1\right)^{N}}{3 L c}
\end{aligned}
$$

and

$$
\begin{aligned}
\left\langle P^{*}\right\rangle_{N+\frac{1}{2}} & =\langle f V\rangle_{N+\frac{1}{2}}=\langle f\rangle_{N+\frac{1}{2}}\left[\left(\langle V\rangle_{N}+\langle V\rangle_{N+1}\right) / 2\right] \\
& =\frac{M|V|^{3}(F-R)^{2}(F+R-1)^{N}\left[2-(F+R)(F+R-1)^{N}\right]}{2 L(2-F-R)} \\
& =\frac{8 M|V|^{5} A^{2} e^{-A}\left(2 e^{-A}-1\right)^{N}\left[1-e^{-A}\left(2 e^{-A}-1\right)^{N}\right]}{9 L c^{2}\left(e^{A}-1\right)} .
\end{aligned}
$$


The second step of (11) is justified because $\left(\langle V\rangle_{N}+\langle V\rangle_{N+1}\right) / 2$ is independent of whether the DP happens to be in state $>+,>-,<+$, or $<-$ at the $N \longrightarrow N+1$ transition, i.e., at the $(N+1)$ st pawl-peg interaction [15].

Time evolution towards final steady state is monotonic-asymptotic (except for $\left\langle P^{*}\right\rangle_{N+\frac{1}{2}}$ ) if $0<$ $F+R-1<1 \Longrightarrow \ln 2>A>0$, diminishing-oscillatory if $-1<F+R-1<0 \Longrightarrow \infty>A>\ln 2$, and complete at $N=1$ if $F+R-1=0 \Longrightarrow A=\ln 2$ [16].

Maxima are: $\langle V\rangle_{N, \max }=\langle V\rangle_{\infty}\left|(A \longrightarrow 0)=2 V^{2} / 3 c,\right| P(V)_{N}-\left.P(V)_{0}\right|_{\max }=\mid P(V \mid A \longrightarrow$ $0)_{\infty}-P(V)_{0}\left|=2 P(V)_{0}\right| V\left|/ 3 c,\langle f\rangle_{N+\frac{1}{2}, \max }=\langle f\rangle_{\frac{1}{2}}\right|(A=1)=4 M|V|^{3} / 3 e c L$, and $\left\langle P^{*}\right\rangle_{\frac{1}{2}, \max }=$ $\left.\left\langle P^{*}\right\rangle_{\frac{1}{2}}|(A=1)=8 M| V\right|^{5} /\left[(3 e c)^{2} L\right]$. Equal and/or higher maxima - if any exist - of $\left\langle P^{*}\right\rangle_{N+\frac{1}{2}}$ for $N \geq 1$ (corresponding to optima of $A$ in the range $0<A<\ln 2$ ) can be found numerically [16].

$\langle V\rangle_{N},\langle f\rangle_{N+\frac{1}{2}}$, and $\left\langle P^{*}\right\rangle_{N+\frac{1}{2}}$ are defined for a $\pm|V|$ pair [17]: hence, considering all $|V|$ and averaging $V^{2},|V|^{3}$, and $|V|^{5}$ in the respective last terms of (8), (10), and (11) over $P(|V|)_{\mathrm{mw}}[17,18]$ yields $\left\langle\left\langle V^{2}\right\rangle\right\rangle_{\mathrm{mw}}=k T / M,\left\langle\left\langle|V|^{3}\right\rangle\right\rangle_{\mathrm{mw}}=\left[2(k T / M)^{3}\right]^{1 / 2}$, and $\left\langle\left\langle|V|^{5}\right\rangle\right\rangle_{\mathrm{mw}}=8\left[2(k T / M)^{5}\right]^{1 / 2}$, respectively; thence, the respective expectation values $\langle\langle V\rangle\rangle_{N},\langle\langle f\rangle\rangle_{N+\frac{1}{2}}$, and $\left\langle\left\langle P^{*}\right\rangle\right\rangle_{N+\frac{1}{2}}$ over all $|V|[17]-$ and, via the immediately preceding paragraph, the respective maxima thereof $\langle\langle V\rangle\rangle_{N, \max },\langle\langle f\rangle\rangle_{N+\frac{1}{2}, \max }$, and $\left\langle\left\langle P^{*}\right\rangle\right\rangle_{N+\frac{1}{2} \text {,max }}$. [By (8), the paragraph immediately following (8), and the paragraph containing (9), considering all $|V|$, to first order in $|V| / c, P(|V|)_{N}=P(+|V|)_{N}+P(-|V|)_{N}=P(|V|)_{0}=P(|V|)_{\mathrm{mw}}$; hence, to first order in $|V| / c$, any average $\langle\langle Q\rangle\rangle_{N}$ [17] over $P(|V|)_{\text {mw }}$ equals that over $P(|V|)_{N}$ itself [19].]

Letting $S$ be total entropy, the second law is challenged by

$$
\left\langle\left\langle P^{*}\right\rangle\right\rangle_{N+\frac{1}{2}}>0 \Longrightarrow d S / d t=-\left\langle\left\langle P^{*}\right\rangle\right\rangle_{N+\frac{1}{2}} / T<0
$$

with maximum challenge if $\left\langle\left\langle P^{*}\right\rangle\right\rangle_{N+\frac{1}{2}}=\left\langle\left\langle P^{*}\right\rangle\right\rangle_{N+\frac{1}{2} \text {,max }}$ (given optimized $A$ ).

Perhaps, DP performance may be improved if a nonrelativistic nonzero rest-mass thermal background medium is preponderant over the EBR [20].

In a longer paper [11b], more thorough analyses are given.

\section{Acknowledgments}

Dr. Donald H. Kobe is gratefully acknowledged for very helpful and extensive discussions and correspondences. I thank Dr. Paolo Grigolini for supplementary and background discussions. I am grateful to Dr. Daniel P. Sheehan for a draft of relevant material from his new book [4c], as well as for the assistance of Drs. Alexey V. Nikulov and Michel Petitjean. Mr. S. Mort Zimmerman is thanked for assistance with proofreading. The referee's report has been constructive and helpful.

\section{Appendix: DP — especially pawl - (re)thermalization}

It has been shown [11] that, for any given $V$, the ratio of (a) the time $\Delta t^{\prime}$ typically required for DP-EBR (as opposed to pawl-peg-bounce) $X$-directional momentum exchanges to effect significant $|\Delta V| /\langle\langle|V|\rangle\rangle_{\mathrm{mw}}=|\Delta V|(\pi M / 2 k T)^{1 / 2}$, to (b) the time $\Delta t^{\prime \prime}$, beginning immediately following a pawlpeg bounce and consequent reversal of $\operatorname{sgn} V$, typically required for the $\pm X$ disk faces to (re)thermalize 
(i.e., for reversal of $\operatorname{sgn}\left[T_{ \pm}(V)-T\right]$ ) via DP-EBR $X$-directional thermal-energy exchanges, is $\Delta t^{\prime} / \Delta t^{\prime \prime} \approx$ (DP rest-mass energy $\left.=M c^{2}\right) /\left(\mathrm{DP}\right.$ thermal energy $\left.\approx M C^{*} T=C T\right)$, where $C^{*}\left(C=M C^{*}\right)$ is the DP's specific heat per unit mass (total heat capacity) $[11,21]$. Hence [letting $\langle\langle\Delta t\rangle\rangle_{\text {bounce }}$ be the average (over all $|V|)$ time interval separating consecutive pawl-peg bounces], $\Delta t^{\prime \prime} \ll\langle\langle\Delta t\rangle\rangle=L /\langle\langle|V|\rangle\rangle_{\mathrm{mw}}=$ $L(\pi M / 2 k T)^{1 / 2}<\langle\langle\Delta t\rangle\rangle_{\text {bounce }}=\langle\langle\Delta t\rangle\rangle /\left(1-e^{-A}\right) \ll \Delta t^{\prime}$ can easily obtain [11].

Fluctuations of the pawl's altitude $Z$ obtain mainly via its intermolecular $Z$-directional momentum exchanges with the $+X$ disk face, which is a "local heat bath" [22] at temperature $T_{+}(V)[11,12,22]$ for the pawl when the DP's $X$-directional Brownian-motional velocity happens to be $V$; by comparison, pawl-EBR $Z$-directional momentum exchanges are negligible [22]. For simplicity, let pawl $/+X$ disk-face $Z$-directional momentum exchanges - and hence the pawl's "sampling" of its Boltzmann distribution corresponding to $T_{+}(V)$ as per (3), (4), and (5) - occur mainly at pawl-stop bounces when $Z=Z_{\min }$ : the stop [10] is within the $+X$ disk face and hence part of said "local heat bath" [22] at temperature $T_{+}(V)[11,12,22] . A \equiv m g H_{\text {net }} / k T$ can easily be large enough so that $\langle\langle\Delta t\rangle\rangle_{\text {bounce }}=$ $\langle\langle\Delta t\rangle\rangle /\left(1-e^{-A}\right) \ll \Delta t^{\prime} \Longrightarrow\langle\langle\Delta t\rangle\rangle \ll\left(1-e^{-A}\right) \Delta t^{\prime} \approx \Delta t^{\prime}$, yet small enough so that $H_{\text {net }} \ll L$ [11]. Let $v$ be the pawl's (nonrelativistic) $Z$-directional Brownian-motional velocity. Averaging over $v$ 's one-dimensional Maxwellian probability density $P(v)_{\mathrm{mw}}=(m / 2 \pi k T)^{1 / 2} \exp \left(-m v^{2} / 2 k T\right)$ yields $\langle|v|\rangle_{\mathrm{mw}}=(2 k T / \pi m)^{1 / 2}$ [23] — which, of course, exceeds $\langle\langle|V|\rangle\rangle_{\mathrm{mw}}=(2 k T / \pi M)^{1 / 2}$ by the ratio $(M / m)^{1 / 2}$. Let $\Delta t^{\prime \prime \prime}=H_{\text {net }} /|v|$ : its average is $\left\langle\Delta t^{\prime \prime \prime}\right\rangle=H_{\text {net }} /\langle|v|\rangle_{\text {mw }}=H_{\text {net }}(\pi m / 2 k T)^{1 / 2}$ [23]. Hence, $\left\langle\Delta t^{\prime \prime \prime}\right\rangle /\langle\langle\Delta t\rangle\rangle=\left(H_{\text {net }} / L\right)(m / M)^{1 / 2}$, which is $\ll 1$ given $H_{\text {net }} \ll L$ and $m \ll M$.

Hence, the pawl is a one-Brownian-particle "isothermal atmosphere" in local TEQ [11,22] at temperature $T_{+}(V)[11,12,22]$ when the DP's $X$-directional Brownian-motional velocity happens to be $V$.

\section{References and Notes}

1. (a) Zhang, K.; Zhang, K. Mechanical Models of Maxwell's Demon with Noninvariant Phase Volume. Phys. Rev. A 1992, 46, 4598-4605. [Ref. [1a] is a classical rather than a quantum-mechanical treatment; as is true of this present paper, Ref. [9], Ref. [3g], and Ref. [11b] (in preparation); but, in contrast therewith, Ref. [1a] considers only velocity-dependent forces that are perpendicular to the velocity (i.e., to the direction of motion), and hence which can do no work.] Ref. [1a] was partially anticipated earlier by:

(b) Bridgman, P. W. Note on the Principle of Detailed Balancing. Phys. Rev. 1928, 31, 101-102; and, in extension, by

(c) Davies, P. C. W. The Physics of Time Asymmetry. University of California Press: Berkeley, 1974; Sec. 6.4 (especially the last paragraph).

2. Allahverdyan, A. E.; Nieuwenhuizen, Th. M. A mathematical theorem as the basis for the second law: Thomson's formulation applied to equilibrium. Physica A 2002, 305, 542-552. See the first and fifth paragraphs of Sec. 5.

3. Feynman's classic "Ratchet and pawl" chapter is:

(a) Feynman, R. P.; Leighton, R. B.; Sands, M. The Feynman Lectures on Physics. Addison Wesley: Reading, Mass, 1963 (Commemorative Issue. Addison Wesley: Redwood City, Calif., 1989); Vol. 1, Chap. 46. In Secs. 46-1 and 46-2, Feynman's classic ratchet and pawl per se is discussed, with the upshot as the last paragraph of Sec. 46-2: "In spite of all our cleverness of lopsided design, if the two temperatures are exactly equal there is no more propensity to turn one way than the other. The 
moment we look at it, it may be turning one way or the other, but in the long run it gets nowhere. The fact that it gets nowhere is really the fundamental deep principle on which all of thermodynamics is based." This upshot is qualitatively justified on bases of (i) mechanics in Sec. 46-3 and (ii) special initial conditions in Secs. 46-4 and 46-5.

More recent analyses of Feynman's classic system are developed in, e.g.:

(b) Parrondo, J. M. R.; Español, P. Criticism of Feynman's analysis of the ratchet as an engine. Am. J. Phys. 1996, 64, 1125-1130.

(c) Magnasco, M. O.; Stolovitzky, G. Feynman's Ratchet and Pawl. J. Stat. Phys. 1998, 93, 615-632.

(d) Jarzynski, C.; Mazonka, O. Feynman's ratchet and pawl: An exactly solvable model. Phys. Rev.

E 1999, 59, 6448-6454.

(e) Velasco, S.; Roco, J. M. M.; Medina, A.; Hernandez, A. C. Feynman's Ratchet optimization: maximum power and maximum efficiency regimes. J. Phys. D: Appl. Phys. 2001, 34, 1000-1006.

(f) Pesz, K.; Gabrys, B. J.; Bartkiewicz, S. J. Analytical solution for the Feynman ratchet. Phys. Rev. E 2002, 66, 061103.

For a survey (as of 1989) anticipating our velocity-dependent DP, see:

(g) Denur, J. Velocity-dependent fluctuations: Breaking the randomness of Brownian motion. Phys. Rev. A 1989, 40, 5390-5399. Erratum. Phys. Rev. A 1990, 41, 3390, Sec. I and Footnotes 1-5.

4. Investigations of various formulations of the second law, various viewpoints concerning the absolute validity thereof, and many challenges thereto are investigated in

(a) Sheehan, D. P., Editor. First International Conference on Quantum Limits to the Second Law, AIP Conference Proceedings 643. AIP: New York, 2002. Various authors cited in Footnotes [5] [8] also have papers published in this AIP Conference Proceedings 643. See also

(b) Sheehan, D. P. Challenges to the Second Law of Thermodynamics (in the Fundamental Theories of Physics Series). Kluwer Academic: New York, estimated publication date 2004.

(c) Sheehan, D. P. A draft of the section of Ref. [4b] that discusses Ref. [3g] has been helpful.

5. Viewpoints concerning the second law in systems manifesting quantum-mechanical entanglement and/or coherence range from (I) that it can be violated, e.g.:

(a) Ćápek, V.; Bok, J. Violation of the second law of thermodynamics in the quantum microworld. Physica A 2001, 290, 379-401.

(b) Cápek, V.; Frege, O. Violation of the 2nd law of thermodynamics in the quantum microworld. Czech. J. Phys. 2002, 52, 679-694.

(c) Ćápek, V. Zeroth and second laws of thermodynamics simultaneously questioned in the quantum microworld. Eur. Phys. J. B 2002, 25, 101-113.

(d) Allahverdyan, A. E.; Nieuwenhuizen, Th. M. Breakdown of the Landauer bound for information erasure in the quantum regime. Phys. Rev. E 2001, 64, 056117.

(e) Allahverdyan, A. E.; Nieuwenhuizen, Th. M. Statistical thermodynamics of quantum Brownian motion: Construction of perpetuum mobile of the second kind. Phys. Rev. E 2002, 66, 036102.

(f) Cápek, V. Dimer as a challenge to the second law. Eur. Phys. J. B 2003, 34, 219-223.

(g) Nieuwenhuizen, Th. M. Thermodynamics and small quantum systems. J. Mod. Optics 2003, 50, 2433-2441.

to (II) that it cannot, e.g.:

(h) Scully, M. O. Extracting Work from a Single Thermal bath via Quantum Negentropy. Phys. Rev. Lett. 2001, 87, 220601.

(i) Hoffmann, K. H. Quantum thermodynamics. Ann. der Phys. 2001, 10, 79-88. 
(j) Novotny, T. Investigation of apparent violation of the second law of thermodynamics in quantum transport studies. Europhys. Lett. 2002, 59, 648-654.

(k) Linke, H. Coherent power booster. Science 2003, 299, 841-842.

(1) Scully, M. O.; Zubairy, M. S.; Agarwal, G. S.; Walther, H. Extracting work from a single heat bath via vanishing quantum coherence. Science 2003, 299, 862-864.

A qualitative summary is given in:

(m) Weiss, P. Breaking the Law. Science News Oct. 7, 2000, 158 (No. 15), pp. 234, 235, and 239.

For very recent studies concerning the quantum-mechanical second law, see:

(n) Allahverdyan, A. E.; Balian, R; Nieuwenhuizen, Th. M. Quantum thermodynamics: thermodynamics at the nanoscale. arXiv:cond-mat/0402387v1 14 Feb 2004, and Refs. 6 and 14 cited therein. Note: Ref. [5f] was Dr. V. Čápek’s last paper before he passed away. Therein, Dr. V. Č́apek responds to Ref. [5j]. Also, in Ref. [5f], Dr. Daniel P. Sheehan has written a tribute to Dr. V. Č́aek.

6. Spontaneous rectification of thermal voltage fluctuations in diodes with very small capacitance at very low temperatures is studied in, e.g.:

(a) McFee, R. Self-Rectification in Diodes and the Second Law of Thermodynamics. Am. J. Phys. 1971, 39, 814-820.

Spontaneous rectification based on the Little-Parks effect is investigated in, e.g.:

(b) Nikulov, A. V. Quantum force in a superconductor. Phys. Rev. B 2001, 64, 012505.

(c) Nikulov, A. V. One of Possible Applications of High-Tc Superconductors. arXiv:physics/0106020 v1 6 Jun 2001.

(d) Nikulov, A. About Perpetuum Mobile without Emotions. Ref. [4a], pp. 207-212.

(e) Dubonos, S. V.; Kuznetsov, V. I.; Zhilyaev, I. N.; Nikulov, A. V.; Firsov, A. A. Observation of the external-ac-current-induced dc voltage proportional to the steady current in superconducting loops. JETP Lett. 2003, 77, 371-375.

(f) Arsitov, A. A.; Nikulov, A. V. Quantum Power Source. Putting in Order of a Brownian Motion without Maxwell's Demon. arXiv:cond-mat/0310073 v1 3 Oct 2003. In Proceedings of SPIE 2003, Vol. 5128 "Quantum informatics”, 148-156.

(g) Berger, J. Noise rectification by superconducting loop with two weak links. arXiv:condmat/0305130 v3 26 Nov 2003.

Spontaneous rectification in nanoscale RLC circuits is studied in, e.g.:

(h) Allahverdyan, A. E.; Nieuwenhuizen, Th. M. Testing the violation of the Claussius inequality in nanoscale electric circuits. Phys. Rev. B 2002, 66, 115309.

7. Proposed violations of the second law in plasma systems can be studied classically, but quantummechanical analyses may be more rigorous. See, e.g., Čápek, V.; Sheehan, D. P. Quantum mechanical model of a plasma system: a challenge to the second law of thermodynamics. Physica $A$ 2002, 304, 461-479.

8. Two examples of the many types of proposed violations of the second law in classical systems obtain: (I) in low-density gas systems in gravitational fields: see, e.g.:

(a) Sheehan, D. P.; Glick, J.; Duncan, T.; Langton, J. A.; Gagliardi, M. J.; Tobe, R. Phase Space

Portraits of an Unresolved Gravitational Maxwell Demon. Found. Phys. 2002, 32, 441-462.

(II) in certain solid-state systems: see, e.g.:

(b) Sheehan, D. P.; Putnam, A. R.; Wright, J. H. A solid-state Maxwell demon. Found. Phys. 2002, 32, 1557-1595.

A concise summary concerning Ref. [8b] is given in: 
(c) Weiss, P. Motor design flouts physical law. Science News Nov. 2, 2002, 162 (No.18), 286. These examples represent only two of many types of proposed violations of the second law in classical systems: see, e.g., Refs. [4a] and [4b] for additional works.

9. This present paper is a revised version of Denur, J. Modified Feynman ratchet with velocitydependent fluctuations. Ref. [4a], pp. 326-331. Ref. [11b] (in preparation) is more detailed.

10. A simple design for the stop: Let the vertical groove that accommodates the pawl have thinner slots extending in the $+Y$ and $-Y$ directions. These slots accommodate pins extending from the pawl in the $+Y$ and $-Y$ directions, respectively. The floors of these slots prevent $Z$-directional motion of the pins below the pin/slot-floor contact level, thereby restricting the pawl's altitude to $Z \geq Z_{\min }$.

11. (a) Ref. [3g]. Sec. IIA and the first two paragraphs of Sec. IIIC supplement the description of our system in the paragraph immediately following Fig. 1 of this present paper. Typical DP-EBR $X$-directional momentum-exchange and DP (re)thermalization timescales are discussed in the two paragraphs immediately following that containing Eq. (7) and in Appendix B, with supplementary material: (i) concerning temperature fluctuations in Appendixes $\mathrm{A}$ and $\mathrm{C}$ and in Footnote 7, and (ii) concerning DP opacity in the two paragraphs immediately following that containing Eq. (23) and the third paragraph of Appendix D. Ref. [9] and this present paper are successive quantitatively improved summaries of Ref. [3g], and Ref. [11b] (in preparation) provides improved detailed discussions.

(b) Denur, J. Time evolution of a modified Feynman ratchet with velocity-dependent fluctuations and the second law of thermodynamics (in preparation). http://www.ipmt-hpm.ac.ru/SecondLaw 2004. Regarding (i) [(ii)] in [11a], see Appendixes B, C, and I [Sec. V and Appendix I].

(c) Except when the pawl's undersurface protrudes below the disk, the $+X$ disk face shields it from EBR impinging on the DP from directions with $-X$ components - and, in any case, its surface area is negligible compared with that of the entire pawl.

(d) Consistently with the fourth-to-the-last sentence (especially the last clause thereof) of the paragraph immediately following Fig.1: The combined pawl-plus-peg $X$-directional thickness is $\ll L$; hence, the $X$-directional spatial, and temporal, intervals separating consecutive pawl-over-peg jumps are only negligibly greater [by said thickness, and (said thickness) $/|V|$, respectively] than those separating consecutive pawl-peg bounces (jump preceded or followed by bounce being the intermediate case). More details are given in Appendix A of Ref. [11b].

[Note: For constructive criticisms of an earlier paper,

(e) Denur, J. The Doppler demon. Am. J. Phys. 1981, 49, 352-355, see:

(f) Motz, H. The Doppler demon exorcised. Am. J. Phys. 1983, 51, 72-73.

(g) Chardin, G. No free lunch for the Doppler demon. Am. J. Phys. 1984, 52, 252-253.

Hopefully, these constructive criticisms have been addressed in Ref. [3g]; with further successive improvements (as noted in [11a]) in Ref. [9], this present paper, and Ref. [11b] (in preparation).]

12. See, e.g.:

(a) Peebles, P. J. E. Principles of Physical Cosmology. Princeton University Press: Princeton, N. J., 1993; pp. 151-158, 174, and 176-181.

(b) Misner, C. W., Kip S. Thorne, K. S.; Wheeler, J. A. Gravitation. W. H. Freeman: New York, 1973; Sec. 22.6 [especially pp. 587-589 and most especially Exercise 22.17 (of Chap. 22) on pp. 588-589].

(c) Peebles, P. J. E.; Wilkinson, D. T. Comment on the Anisotropy of the Primeval Fireball. Phys. Rev. 1968, 174, 2168.

13. See, e.g.: 
(a) Kolmogorov, A. N. Foundations of the Theory of Probability, Second English Edition. Chelsea: New York, 1956.

(b) Ghahramani, S. Fundamentals of Probability, Second Edition. Prentice Hall: Upper Saddle River, N. J., 2000.

(c) Kelly, D. G. Introduction to Probability. Macmillan: New York, 1994.

14. See, e.g.:

(a) Cox, D. R.; Miller, H. D. The Theory of Stochastic Processes. Chapman and Hall: London, 1965 (1990 Printing); Secs. 3.1, 3.2, and 3.6.

(b) Hoel, P. G.; Port, S. C.; Stone, C. J. Introduction to Stochastic Processes. Houghton Mifflin: Boston, 1972 (reissued: Waveland: Prospect Heights, Ill., 1987); pp. 1-2, Secs. 1.1, 1.2, and 1.4.2, and pp. 47-49.

15. Ref. [6c], Sec. 4.2.; Ref. [6d], pp. 209-210; Ref. [11b] (in preparation), Eq. (18) and the two immediately following sentences, and Appendix D.

16. More details are given in Ref. [11b], especially in Sec. IV thereof.

17. Any $\langle Q\rangle_{N}$, e.g., $\langle V\rangle_{N},\langle f\rangle_{N+\frac{1}{2}}$, or $\left\langle P^{*}\right\rangle_{N+\frac{1}{2}}$, is defined given a $\pm|V|$ pair, i.e., given $|V|-$ it is undefined and cannot even be calculated given only a single value of $V$, e.g., given only $+|V|$ alone or given only $-|V|$ alone. $\langle Q\rangle_{N}$ can be written in the more detailed form $\langle Q(|V|)\rangle_{N}$; by contrast, the expression $\langle Q(V)\rangle_{N}$ is meaningless. Averaging over any one given $\pm|V|$ pair to obtain $\langle Q\rangle_{N}$ first, and subsequently averaging over all $|V|$ to obtain $\langle\langle Q\rangle\rangle_{N}$, is preferable to attempting to obtain $\langle\langle Q\rangle\rangle_{N}$ directly because, e.g.: (a) the former procedure is easier, (b) both $\langle Q\rangle_{N}$ and $\langle\langle Q\rangle\rangle_{N}$ are thus obtained, and (c) the $|V|$-dependence of $F-R$ in (8), (10), and (11) is thus accounted for via application of (4) and (5) in the respective last steps thereof - before averaging over all $|V|$ as per the second paragraph and fourth-to-the-last paragraph of Sec. 2.

18. Averaging over $P(V)_{\mathrm{mw}}$ or over $P(|V|)_{\mathrm{mw}}$ obviously yields identical results numerically, but, as per Footnote [17], the latter averages are more correct conceptually.

19. Of course, initially, at $N=0, P(|V|)_{N}=P(|V|)_{0}=P(|V|)_{\mathrm{mw}}$ exactly (not merely to first order in $|V| / c)$; hence, any average $\langle\langle Q\rangle\rangle_{0}$ over $P(|V|)_{\mathrm{mw}}$ is exact.

20. Ref. [3g], Appendix D; Ref. [11b] (in preparation), Appendixes G, H, and I.

21. Both $\Delta t^{\prime}$ and $\Delta t^{\prime \prime}$ considered individually — not merely their ratio - are independent of $V$ to first order in $V / c$. See Ref. [3g], Appendix B; and Ref. [11b] (in preparation), Appendixes B and I.

22. Of course, Doppler-shifted EBR at temperature $T_{+}(V)$ [12] is the primary element of said "local heat bath", with said temperature $T_{+}(V)$ then obtaining for the $+X$ disk face (including the stop and the pawl) itself [11] via $+X$-disk-face/EBR $X$-directional thermal-energy exchanges when the DP's $X$-directional Brownian-motional velocity happens to be $V$. But pawl-EBR $Z$-directional momentum exchanges are negligible compared with pawl/ $+X$-disk-face - specifically, pawl-stop $-Z$ directional momentum exchanges.

23. Enclosure within single angular brackets denoting averaging over all $v$ should not be confused with denotation of averages over any one given $\pm|V|$ pair (over all $|V|$ ) via enclosure within single (double) angular brackets. Recall the second paragraph and fourth-to-the-last paragraph of Sec. 2, and Footnote [17]. (Negligible error results from employing $T$, rather than $T_{+}(V)$, in $P(v)_{\mathrm{mw}}$.)

(c) 2004 by MDPI (http://www.mdpi.org). Reproduction for noncommercial purposes permitted. 